(2), Gray Partridge (1), Sandhill Crane (2), Sora (1), Yellow Rail (3). American Coot (4), Semipalmated Plover (2), Killdeer (11), Common Snipe (2), Long-billed Curlew (1), Sipotted Sandpiper (2), Baird's Sandpiper (1), American Avocet (2: col. 14), Northern Phalarape (1), Herring Gull (3), Ring-billed Gull (1: col. 20), Mew Gull (2), Bonaparte's Gull (1: col. 3). Common Tern (1), Arctic Tern (9: col. 5), Black Tern (9), Rock Dove (2: large col.), Mourning Dove (10), Black-billed Cuckoo (2), Great Horned Owl (14), Burrowing Owl (2), Long-eared Owl (5), Belted-Kingfisher (1), Yellow-shafted Flicker (1), Pileated Woodpecker (1), Downy Woodpecker (3), Eastern Kingbird (9), Western Kingbird (8), Eastern Phoebe (16), Say's Phoebe (5), Least Flycatcher (2), Horned Lark (5), Tree Swallow (10), Bank Swallow (2), Barn Swallow (26). Cliff Swallow (8), Purple Martin (1: col. 42), Blue Jay (1), Black-billed Magpie (8), Common Crow (17), Dipper (1), House Wren (12), Longbilled Marsh Wren (1), Catbird (6), Brown
Throsher (4), Robin (43), Hermit Thrush (1), Gray-cheeked Thrush (2), Veery (2), Mountain Bluebird (4); Townsend's Solitaire (1), Goldencrowned Kinglet (1), Water Pipit (1), Siprague's Pipit (4), Cedar Waxwing (4), Loggerhead Shrike (2), Storling (1), Red-eyed Vireo (2), Warbling Vireo (2), Yellow Warbler, (4), MacGillivary's Warbler (1), Yellowthroat (1), House Sparrow (2), Bobolink (3), Western Meadowlark (8), Yellow-headed Blackbird (6), Red-winged Blackbird (22), Baltimore Oriole (2), Bullock's Oriole (1), Brewer's Blackbird (8), Common Grackle (3), Rose-breasted Grosbeak (3), Hoary Redpoll (5), Common Redipoll (5), American Goldfinch (2), Savannah Sparrow (3), Grasshopper Sparrow (1), Baird's Sparrow (1) Sharp-tailed Sparrow (1), Vesper Sparrow (15), Tree Sparrow (1), Chipping Sparrow (4), Clay-colored Sparrow (8), Harris' Sparrow (3), White-crowned Sparrow (1), Lincoln's Sparrow (1), Song Sparrow (5), McCown's Longspur (1), Lapland Longspur (5), Chestnut-collared Longspur (1).

\title{
Twenty-first Annual Saskatchewan Christmas Bird Count, 1962
}

\section{Edited by Margaret Belcher and Dorothy R. Wade, Regina}

A new record of total species counted was made this year with 68 species reported on count day from 26 localities. Observers recruited for the count numbered 135, with Saskatoon placing the largest number in the field. Even with two guests from Indiana, the Saskatoon total of 36 did not quite reach last year's record of 37 observers from that club.

The total number of species observed in Saskatchewan during 21 years of Christmas counts now stands at 108, the Regina group having added two new species-Ross' Goose and Shoveler.

Late migrants appeared more numerous than usual, presumably owing to the continuing mild weather. Mourning Dove, Brown Creeper, Western Meadowlark, three species of blackbirds and Common Grackle, Slate-colored Junco, Tree Sparrow and Song Sparrow were all reported in one or more localities. Short-eared Owls were more common than usual, reported from 10 points. Thirteen points reported Horned Larks, which were not only present in more localities but were noted frequently in flocks as though forming part of a last wave of migrants.

Gray Partridge were counted in 14 districts, with several compilers com- menting on their numbers and the large size of the winter flocks.

In assembling these records during the absence of Mary and Stuart Houston, we have appreciated the assistance given by Lucy Murray and Douglas Wade.

BATTLEFORD, Sask. Dec. $31 ; 27$ miles by car and 5 miles on foot in $41 / 2$ hours; temp. $-20^{\circ}$; wind $\mathrm{N}$. at $20 \mathrm{mph} ; 10$ inches of snow. 14 species, 218 individuals. Ruffed Grouse, 1; Sharp-tailed Grouse, 1; Rock Dove, 17; Snowy Owl, 1; Hairy Woodpecker, 3; Downy Woodpecker, 2; Blackbilled Magpie, 5; Black-capped Chickadee, 5; Bohemian Waxwing, 52; House Sparrow, 41; Evening Grosbeak, 18; Pine Grosbeak, 16; Common Redpoll, 19; Snow Bunting, 37.-Spencer Sealy.

BLADWORTH, Sask. Dec. 27; 4 hours in the field- $11 / 2$ hours by team, $21 / 2$ hours by truck or. on foot; temp. $10^{\circ}$ to $13^{\circ}$; wind S.W. at 6-8 m.p.h.; clear; snow 6 to 8 inches, mostly in drifts. 8 species, 2195 individuals. Sharp-tailed Grouse, 15; Gray Partridge, 61; Great Horned Owl, 1; Short-eared Owl, 1; Horned Lark, 29; Black-billed Magpie, 10; House Sparrow, 78; Snow Bunting, $2000 \pm$. (Add: Golden Eagle, 2, Dec. 21, Dec. 31; Rock Dove, 8, Dec. 21; 
Starling, 1, Dec. 22).-Bill, Theresa and Lawrence Beckie.

CARON, Sask. Dec. $30 ; 15$ miles by car in 2 hours; temp. $10^{\circ}$; clear; wind light; snow covering sparse. 4 species, 72 individuals. Gray Partridge, 40; Short-eared Owl, 4; Black-billed Magpie, 8; House Sparrow, 20.-Mr. and Mrs. Cy Knight.

DILKE, Sask. Dec. $31 ; 46$ miles by foot and car in 6 hours; temp. $0^{\circ}$ to $8^{\circ}$; wind N.W. 10-35 m.p.h.; overcast; 3" drifted snow. 11 species, 320 individuals. Pigeon Hawk, 1; Gray Partridge, 18; Great Horned Owl, 1; Short-eared Owl, 1; Hairy Wosdpecker, 1; Downy Woodpecker, 1; Horned Lark, 3; Black-billed Magpie, 16; Black-capped Chickadee, 4; Starling, 2; House Sparrow, 250. (Add: Bohemian Waxwing, 2, Dec. 22; Snow Bunting-very numerous most days of the period).-J. B. Belcher (compiler), Margaret Belcher, $\mathbf{M r}$. and Mrs. S. R. Belcher, Janet McArton.

FOXFORD, Sask. Jan. 1; 6 miles on foot in 3 hours; temp. $-1^{\circ}$; wind E.S.E. at 6-8 m.p.h.; 8 inches of snow. 6 species, 32 individuals. Hairy Woodpecker, 1; Dcwny Woodpecker, 3; Black-billed Magpie, 2; Blackcapped Chickadee, 2; House Sparrow, 16; Evening Grosbeak, 8. (Add. Common Raven, 1, Dec. 28; Snow Bunting, 20, Dec. 26).-Tom Bird.

HAWARDEN, Sask. Dec. 31; 20 miles by car and arcund the farm for 6 hours; temp. $10^{\circ}$; partly cloudy; 6 inches snow. 4 species, 227 individuals. Golden Eagle, 1; Gray Partridge, 12; Rock Dove, 14; House Sparrow, 200. (Add: Short-eared Owl, 1, Dec. 27; Horned Lark, 4, Dec. 24).-Harold Kvinge.

HIGH HILL-KLOGEI LAKE, Sa'sk. Dec. $30 ; 6$ hcurs on foot through 9 miles of heavy brush and lakes, and 6 miles by car past fields and poplar bluffs; temp. $5^{\circ}$ to $10^{\circ}$; wind S.E. at 8-10 m.p.h.; cloudy with snow flurries. 12 species, 64 individuals. Great Horned Owl, 1; Hairy Woodpecker, 1; Downy Woodpecker, 1; Black-backed Three-toed Woodpecker, 1; Northern Three-toed Woodpecker, 1; Gray Jay, 3; Black-billed Magpie, 1; Common Raven, 3; Black-capped Chickadee, 12; Evening Grosbeak, 3; Common
Redpoll, 12; Snow Bunting, 25.Anton and Steve Waycheshen.

KINDERSLEY, Sask. Jan. 1; 41 miles by car and 3 miles on foot in 4 hours; temp. $13^{\circ}$; light intermittent snow; 2 inches snow. 11 species, 831 individuals. Prairie Falcon, 1; Gray Partridge, 8; Rock Dove, 20; Great Horned Owl, 1; Short-eared Owl, 3; Horned Lark, 3; Black-billed Magpie, 2; Bohemian Waxwing, 27; Starling, 1; House Sparrow, 732; Snow Bunting, 33. (Add: Sharp-tailed Grouse, 2, Dec. 30; Snowy Owl, 5, Dec. 30).Glen A. Fox (compiler), R. A. and Kim Lamont.

LADY LAKE, Sask. Dec. 23; 2 hours on foot; temp. $10^{\circ}$; clear and calm; 2 inches snow. 7 species, 48 individuals. Downy Woodpecker, 1; Gray Jay, 2; Blue Jay, 1; Black-capped Chickadee, 4; House Sparrow, 8; Pine Grosbeak, 2; Common Redpoll, 30. (add: Ruffed Grouse, 3, Jan. 1; Gray Partridge, 6, Dec. 29 Hairy Woodpecker, 1, Dec. 26; Black-billed Magpie, 1, Jan. 1; Common Raven, 1, Jan. 1.).-Donald J. Buckle.

LEADER, Sask. Dec.; 3 hours afield around farmyard and on a 2-mile walk; temp. $10^{\circ}$; clear and calm; $1 / 2$ inch snow. 4 species, 76 individuals. Ring-necked Pheasant, 5; Downy Woodpecker, 1; Black-billed Magpie, 10; House Sparrow, 60. (Add: Bohemian Waxwing, 5, Dec. 28.-Daisy D. Myers.

ESTEVAN, Sask. Dec. 27; 12 miles by foot and 30 by cer in 8 hours; temp. $15^{\circ}$; wind S.E. at 15 m.p.h.; 4 inches snow on ground; light snow in a.m., clearing in p.m. 23 species, 778 individuals. Mallard, 220; Pintail, 2; Common Goldeneye, 2; Common Merganser, 3; Sharp-tailed Grouse, 2; Ring-necked Pheasant, 1; Rock Dove, 16; Great Horned Owl, 1; Hairy Woodpecker, 1; Horned Lark, 4; Black-billed Magpie, 11; Blackcapped Chickadee, 6; White-breasted Nuthatch, 1; Golden-crowned Kinglet, 11; Bohemian Waxwing, 32; Starling, 10; House Sparrow, 308; Red-winged Blackbird, 30; Rusty Blackbird, 30; Purple Finch, 2; White-winged Crossbill, 10; Tree Sparrow, 4; Snow Bunting, 95. (Add: Red-breasted Nuthatch, 7, Dec. 19). -Darrel Carlson, Ross Lein (compiler). 
FORT QU'APPELLE, Sask. Dec. 26; temp. $4^{\circ}$; wind light; cloudy; 3 inches snow. 15 species, 189 individuals (summary of reports from individual observers). Ruffed Grouse, 1; Sharptailed Grouse, 2; Rock Dove, 15; Mourning Dove, 1; Great Horned Owl, 1; Downy Woodpecker, 2; Horned Lark, 1; Black-billed Magpie, 26; Black-capped Chickadee, 7; Bohemian Waxwing, 1; House Sparrow, 67; Red-winged Blackbird, 3; Rusty Blackbird, 6; Common Redpoll, 6; Snow Bunting, 50. (Add: Eagle, probably Golden but could be immature Bald, 1, Dec. 29; Snowy Owl, 1, Dec. 20; Hairy Woodpecker, 4, several dates and observers; Blue Jay, 3, several dates and observers; Northern Shrike, 1, on three dates by one observer; Western Meadowlark, 1, Dec. 30; Pine Grosbeak, 24, several dates and three observers).-Nancy Bushell, E. M. Callin (compiler), Richard Carter, Errol Cochrane, Peter Flett, Mr. and Mrs. William Gray, Dr. H. D. Jenner, Richard Nevard, Horace Reed, S. Regan, Jos. Rumancik.

McLEAN, Sask. Jan. 1; birds observed in yard. 6 species, 41 individuals. Great Horned Owl, 1; Hairy Woodpecker, 2; Downy Woodpecker, 2; Black-capped Chickadee, 12; Robin, 1; Bohemian Waxwing, 23. (Add: Ruffed Grouse, 4).--Mrs. Hilda Newton, Mrs. Harold Bray.

MASEFIELD, Sask. Dec. 27; 6 miles by car over open country, 5 miles on foot on the Frenchman River; temp. $30^{\circ}$; calm and bright, but clouding over. 15 species, 394 individuals. Marsh Hawk, 1; Sage Grouse, 11; Ring-necked Pheasant,.5; Short-eared Owl, 1; Horned Lark, 5; Black-billed Magpie, 20; Black-capped Chickadee, 3; Starling, 1; House Sparrow, 300; Western Meadowlark, 1; Red-winged Blackbird, 4; Brewer's (?) Blackbird, 1; Pine Grosbeak, 20; Tree Sparrow, 20; Snow Bunting, 1. (Add: Golden Eagle, 2; Prairie Falcon, 1; Pigeon Hawk, 1; Gray Partridge, 9; Snowy Owl, 1, seen Dec. 20 by Bernard Reader; Long-eared Owl, 3, Dec. 23; Northern Shrike, 1, Dec. 29).-J. David Chandler.

MOOSE JAW, Sask. Dec. 26; 20 party miles by car and 21 by foot in 13 party hours; temp. $11^{\circ}$ to $6^{\circ}$; wind N.N.W. at 29 m.p.h. gusting to 38 with blowing snow till noon; partially cloudy; 3 inches snow on ground. 19 species, 1235 individuals. Ringnecked Pheasant, 24; Gray Partridge, 12; Rock Dove, 144; Great Horned Owl, 4; Snowy Owl, 1; Short-eared Owl, 1; Yellow-shafted Flicker, 1; Downy Woodpecker, 2; Horned Lark, 6; Black-billed Magpie, 115; Common Crow, 5; Black-capped Chickadee, 28; White-breasted Nuthatch, 4; Starling, 7; House Sparrow, 650; Brewer's Blackbird, 2; Pine Grosbeak, 15; Common Redpoll, 10; Snow Bunting, 204. (Add: Pigeon Hawk, 1, Dec. 28; Bohemian Waxwing, 7, Dec. 25; Slate-colored Junco, 8, Dec. 28).-Mrr. and Mrs. Carl Ellis, Mary Jane Ellis, John Ellis and Jack Zess (compilers), Mr. and Mrs. Cy Knight, W. W. Riome, Mrs. C. V. Stokins, Robert Tabaka, Mrs. F. B. Taylor, Hazel Winn (members of Moose Jaw Natural History Society).

PELLY, Sask. Dec. 22; 4 hours by car, one hour on foot; temp. $-5^{\circ}$; wind N.W. at 10-20 m.p.h.; cloudy with sunny intervals and blowing snow; 3 inches fresh snow. 9 species, 194 individuals. Downy Woodpecker, 1; Blue Jay, 2; Black-billed Magpie, 5; Black-capped Chickadee, 3; House Sparrow, 76; Evening Grosbeak, 1; Pine Grosbeak, 10; Common Redpoll, 5; Snow Bunting, 91. (Add: Goshawk, 1, Dec. 26; Sharp-tailed Grouse, 25, Dec. 26; Horned Owl, 1, Dec. 23; Hairy Woodpecker, 8, Dec. 26).-R. and S. Zazelenchuk.

PRINCE ALBERT, Sask. Jan. 2; mixed wood forest, black spruce bog, pine forest. 11 species, 186 individuals. Downy Woodpecker, 2; Black-billed Magpie, 1; Common Raven, 16; Black-capped Chickadee, 6; Boreal Chickadee, 1; Red-breasted Nuthatch, 1; Brown Creeper, 1; Bohemian Waxwing, 120; Evening Grosbeak, 31; Pine Grosbeak, 5; Common Redpoll, 2. IED. NOTE: This count was made on Jan. 2, one day later than the official count period.l -Donald Karasiuk.

REGINA, Sask. Dec. 30； 259 party miles by car and 44 party miles by foot in 147 party hours; temp. $-3^{\circ}$ to $12^{\circ}$; wind S.E. at 20 m.p.h.; cloudy, with blowing snow. 34 species, 5306 individuals. Eared Grebe, 2; Mute Swan, 8; Whistling Swan, 4 (one crippled); Canada Goose, 237; Ross' Goose, 1; Mallard, 502; Blue-winged 
Teal, 2 (one crippled); Shoveler, 1; Lesser Scaup, 10; Common Goldeneye, 1; Ruddy Duck, 7; Golden Eagle, 1; Sharp-tailed Grouse, 11; Ringnecked Pheasant, 18; Gray Partridge, 94; American Coot, 9; Rock Dove, 41; Great Horned Owl, 6; Snowy Owl, 3; Short-eared Owl, 3; Downy Woodpecker, 4; Horned Lark, 17; Blackbilled Magpie, 63; Common Crow, 1; Black-capped Chickadee, 15; Brown Creeper, 2; Bohemian Waxwing, 8; Northern Shrike, 1; Starling, 21; House Sparrow, 3305; Pine Grosbeak, 3; Cammon Redpoll, 2; White-winged Crossbill, 13; Snow Bunting, 890. (Add: Pintail (injured), 1, Dec. 19, 23; American Widgeon, 1, Dec. 19; Canvasback, 1, Dec. 23; Prairie Falcon, 1, Dec. 19; Pigeon Hawk, 1, Dec. 28; Ruffed Grouse, 6, Jan. 1; Golden-crowned Kinglet, 1, Dec. 23; Rusty Blackbird, 1, Dec. 21).-Ron Austin, Margaret Belcher, Frank Brazier (compiler), Betty Cruickshank, Adam Deutscher, Joyce Deutscher, Elmer Fox, Reg Fox, Doug Gilroy, Sylvia Harrison, Bernie Haysom, Ray Knudsen, Fern Lawrence, George Ledingham, Lorraine MacPherson, Robert Nero, Richard Robinson, Allan Smith, Herb Tempel, Ruth Tempel, Alan Wade, Dorothy Wade, Doug Wade, Vic Wilshire (Regina Natural History Society).

ROUND LAKE (six miles north of High Hill, Sask.). Dec. 28; 7 hours on foot through 10 miles of deciduous woods mixed with spruce; temp. $5^{\circ}$; wind N.W. at 10-15 m.p.h.; cloudy with snow flurries. 10 species, 78 individuals. Ruffed Grouse, 3; Sharp-tailed Grouse, 1; Hairy Woodpecker, 1; Downy Woodpecker, 1; Common Raven, 2; Black-capped Chickadee, 1; Boreal Chickadee, 1; House Sparrow, 6; Evening Grosbeak, 2; Common Redpoll, 60 (est.). (Add: Pileated Woodpecker, 1, Dec. 21).Steve Waycheshen.

SASKATOON, Sask. (All points within 15-mile diameter circle, including city, banks of South Saskatchewan River, airport, Forest Experimental Research Station; woodland $5 \%$, mixed farming and pasture $90 \%$, city $5 \%$ ). Dec. $26 ; 8: 30$ a.m to $4: 30$ p.m.; temp. $4^{\circ}$ to $9^{\circ}$; wind N. at 22 to W.N.W. at 7 m.p.h.; 5 inches snow, drifts to 2 feet. Total party hours, 26 $(81 / 2$ on foot, $171 / 2$ by car); total party miles, 219 (21 on foot, 198 by car). 23 species, 4348 individuals. Mallard, 3; Lesser Scaup, 3; Common Goldeneye, 1; Sharp-tailed Grouse, 30; Ring-necked Pheasant, 21; Gray Partridge, 44; Rock Dove, 256; Great Horned Owl, 2; Yellow-shafted Flicker, 2; Hairy Woodpecker, 2; Downy Woodpecker, 1; Blue Jay, 9; Black-billed Magpie, 127; Blackcapped Chickadee, 16; Bohemian Waxwing, 409; Cedar Waxwing, 34; Starling, 4; House Sparrow, 2258; Common Grackle, 2; Pine Grosbeak, 123; Hoary Redpoll, 2; Common Redpoll, 163; Snow Bunting, 836. (Add: Pigeon Hawk, 1, Dec. 31; Snowy Owl, 1, Dec. 27; Horned Lark, 1, Jan. 1; Robin 1 Dec. 19-27).-Harold Belcher, John Black, Pern Cordery, Dr. and Mrs. Ray Denson, Mr. and Mrs. J. C. Finmey (Anderson, Indiana), Bob Folker, George Gerrity, Ross Gerrity, Mr. and Mrs. Verne Gimbel, Ivan Gimbel, Sherry Gimbel, Mr. and Mrs. Bernard Gollop, Gladys Hall, Jim Hamilton, Mr. and Mrs. Jim Hogg, Connie King, Janet McDougall, Greg Michalenko, Bob Mills, Shirley Orr, Bob Pravda, Frank Roy (compiler), John Shadick, Dorothy Slater, Jim Slimmon, Mr. and Mrs. Ron Watts, Ted Wedge, Terry Wedge, Jim Wedgwood, Wendy Wood (members and friends of Saskatoon Natural History Society).

SHAUNAVON, Sask. Dec. $22 ; 41 / 2$ miles on foot along the White Mud River and 65 miles by car; temp. $0^{\circ}$; cloudy; 1 inch snow. 10 species, 671 individuals. Golden Eagle, 1; Peregrine Falcon, 1; Sharp-tailed Grouse, 25; Ring-necked Pheasant, 11; Gray Partridge, 11; Horned Lark, 36; Black-billed Magpie, 113; Starling, 22; House Sparrow, 346; Common Redpoll, 85.-Doug Young.

SKULL CREEK, Sask. Jan. 4, 1963; 3 hours; temp. $46^{\circ}$ to $38^{\circ} ; 11$ species, 139 individuals. Common Merganser, 6; Golden Eagle, 1; Prairie Falcon, 1; Ruffed Grouse, 6; Sharp-tailed Grouse, 5; Ring-necked Pheasant, 11; Gray Partridge, 40; Saw-whet Owl, 2; Downy Woodpecker, 8; Horned Lark, 37; Bohemian Waxwing, 20. (Add: Green-winged Teal, 1, Dec. 20; Sharp-shinned Hawk, 1, Jan. 2; Rough-legged Hawk, 1, Dec. 27; Sage Grouse, 2, Dec. 18; Snowy Owl, 1, Jan. 2; Short-eared Owl, 1, Dec. 
27, Jan. 3; Hairy Woodpecker, 1, Dec. 26; Blue Jay, 1, Dec. 21; Northern Shrike, 1, Dec. 24.) [ED. NOTE: The Skull Creek count was made on Jan. 4, three days later than the official count period.1-Henry, Doug and Mrs. Edith Borman, Carolyn Drever, Mrs. Betty Mann, Steve Mann (compiler), Mrs. Steve Mann, Joan and Fred Stevens, Peter, George and Donna Swain, Harry Williams.

SPIRIT LAKE, Sask. Dec. 30; sandhills, hay meadows, farmyards; 5 party hours and 5 party miles on foot; temp. $-1^{\circ}$; wind S.E. light; snow flurries. 13 species, 143 individuals. Ruffed Grouse, 1; Hairy Woodpecker, 6; Downy Woodpecker, 9; Blue Jay, 1; Black-billed Magpie, 2; Black-capped Chickadee, 60; White-breasted Nuthatch, 2; House Sparrow, 40; Evening Grosbeak, 9; Pine Grosbeak, 2; Common Redpoll, 5; Slate-colored Junco, 1; Snow Bunting, 5. (Add: Great Horned Owl, 1, Dec. 21; Horned Lark, 2, Dec. 28-Jan. 1; Bohemian Waxwing, 2, Dec. 21, Dec. 26, Dec. 31, Jan. 1).-Bill Anaka, Joyce Gunn.

TULLIS, Sask. Dec. 27; open prairie; 3 hours -6 miles by truck, otherwise on foot; temp. $17^{\circ}$; clear, becoming cloudy and colder; little snow. 4 species, 20 individuals. Sharp-tailed Grouse, 2; Black-billed Magpie, 5; House Sparrow, 11; Snow Bunting, 2. (Add: Snowy Owl, 1, Dec. 20; Common Redpoll, 2, Dec. 28).-Mrs. E. C. Boon, Chris and Robert Boon.

WOLSELEY, Sask. Dec. 30; 8 miles on horseback; temp. $8^{\circ}$; wind 15 m.p.h.; mostly clear; 6 inches snow. 8 species, 144 individuals. Ruffed Grouse, 1; Hairy Woodpecker, 1; Downy Woodpecker, 1; Black-billed Magpie, 8; Black-capped Chickadee, 1; House Sparrow, 100; Common Redpoll, 31; Song Sparrow, 1. (Add: Hawk (large hawk unidentified), 1, Dec. 24; Sharp-tailed Grouse, 23, Dec. 21, 26; Gray Partridge, 10, Dec. 20; Great Horned Owl, 1, Dec. 24; Northern Shrike, 1, Dec. 20; Starling, 19, Dec. 21; Pine Grosbeak, 1, Dec. 26; Snow Bunting, 40, Dec. 19).Donald Hayward.

WOODROW, Sask. Dec. 20. 7 species, 252 individuals. Ring-necked Pheasant, 14; Gray Partridge, 31; Snowy Owl, 1; Short-eared Owl, 1; Blackbilled Magpie, 3; Starling, 2; House Sparrow, $200+.-$ C. H. Shulver.

\section{First Manitoba Christmas Bird Count, 1962}

Edited by David Hatch, Oak Lake, Manitoba

This summary covers the first province-wide Christmas Count to be conducted in Manitoba. Thirteen areas took part in the survey, with a co-operative group of seven parties in Winnipeg making the biggest count in the province, of 23 species.

The exceedingly mild fall and winter preceding Christmas day resulted in many species remaining in the area instead of migrating south. The Horned Larks seen at Oak Lake and Melita were unusual for this time of year, but like the Tree Sparrows at Oak Lake and Slate-colored Juncos at Winnipeg and Brandon, had been recorded more than once on counts in past three years. The five Mallards seen in Winnipeg and the 67 Common Goldeneyes from Point du Bois and Seven Sisters were the only waterfowl on the count. One Harris' Sparrow, one Song Sparrow, and two Marsh Hawks in Winnipeg were odd- ities, as was the Sparrow Hawk at Brandon and the Mockingbird at Oak Lake. The count produced only five Snowy Owls. The Barred Owl reported from Point du Bois was an unconfirmed report. Woodpeckers were down sharply with only one Blackbacked Three-toed Woodpecker, 26 Hairy Woodpeckers and 21 Downy Woodpeckers. Evening Grosbeaks, Pine Grosbeaks and Bohemian Waxwings were here in smaller number than in 1961. A total of 45 species was seen in the province during the count period. Dr. Lawrie B. Smith and Mr. Harold V. Hosforth kindly assisted in compiling the counts.

ASHERN, Man. Dec. 30; semi-open country with some black spruce and poplar; 2 miles on foot in one hour, 6 miles by car in $1 / 2$ hour $(3$ p.m. to $4: 30$ p.m.) ; temp. $-10^{\circ}$ to $-5^{\circ}$; clear; 10 inches snow. 7 species, 51 individuals. Ruffed Grouse, 1; Sharp- 